INFLAMMATORY BOWEL DISEASE

\title{
Cholera toxin subunit B inhibits IL-12 and IFN- $\gamma$ production and signaling in experimental colitis and Crohn's disease
}

\author{
E M Coccia, M E Remoli, C Di Giacinto, B Del Zotto, E Giacomini, G Monteleone, \\ M Boirivant
}

See end of article for authors' affiliations

\section{Correspondence to:} $\operatorname{Dr} M$ Boirivant, Department of Infectious, Parasitic and Immunemediated diseases, Istituto Superiore di Sanità, Viale Regina Elena 299, 00161 Rome, Italy; monboir@iss.it

Revised 26 May 2005 Accepted 21 June 2005 Published online first 16 August 2005

\begin{abstract}
Background and aims: Cholera toxin B subunit (CT-B) is a powerful modulator of immune responses. The authors have previously demonstrated that oral administration of recombinant CT-B (rCT-B) is able to prevent and cure the Crohn's disease (CD)-like trinitrobenzene sulfonic acid (TNBS) mediated colitis. In this study they extended their observations and examined if rCT-B interferes with the molecular signaling underlying the Th1 type response both in TNBS colitis and in ex vivo human CD explants.

Methods: TNBS treated mice were fed with $\mathrm{rCT}-\mathrm{B}$, and IFN- $\gamma$ and IL-12 production by colonic lamina propria mononuclear cells (LPMC) was examined by ELISA. In vitro culture of mucosal explants from CD patients and non-inflammatory bowel disease controls, pre-incubated with rCT-B, were examined for IFN$\gamma$ and IL-12 production by ELISA and semiquantitative reverse transcription polymerase chain reactions. STAT-1, - $4,-6$ activation and T-bet expression were examined following $\mathrm{rCT}-\mathrm{B}$ treatment by western blotting both in TNBS treated mice and in human mucosal explants.

Results: $\mathrm{rCT}-\mathrm{B}$ significantly reduced IL-12 and IFN- $\gamma$ secretion by LPMC from TNBS treated mice. Consistent with this, rCT-B inhibited both STAT-4 and STAT-1 activation and downregulated T-bet expression. Inhibition of Th1 signaling by CT-B associated with no change in IL- 4 synthesis and expression of active STAT-6 indicating that $r C T-B$ does not enhance Th2 cell responses. Moreover, in vitro treatment of CD mucosal explants with $r C T-B$ resulted in reduced secretion of IL-12/IFN- $\gamma$ and inhibition of STAT-4/STAT- 1 activation and T-bet expression.

Conclusions: These studies indicate that CT-B inhibits mucosal Th1 cell signaling and suggest that $\mathrm{rCT}-\mathrm{B}$ may be a promising candidate for $C D$ therapy.
\end{abstract}

t nflammatory bowel diseases (IBD) denote chronic inflammatory disorders of gastrointestinal tract that comprises two major groups: Crohn's disease (CD) and ulcerative colitis (UC). ${ }^{1}$ The aetiology of IBD remains unknown, but there is evidence that a dysregulated immune reactivity against luminal bacteria plays a major role in the induction and perpetuation of the ongoing inflammation. ${ }^{2} \mathrm{In} C D$, this is associated with aberrant production of Thl cytokines and activation of Thl transcription factors. Indeed, increased production of IFN- $\gamma$ and IL- $12^{3-6}$ is seen in CD tissue, and selective downmodulation of Thl type inflammation appears to be an optimal way to achieve stable remission in these patients.

Cholera toxin (CT) is composed of a monomeric A subunit (CT-A) associated with a pentameric B subunit (CT-B). While the former is responsible for the secretory effects of the toxin, the latter is necessary for both binding to cell surface monosialogangliosides and facilitation of toxin entry. In this form CT is a potent immunogen and mucosal adjuvant, capable of stimulating antigen specific secretory IgA and systemic IgG antibody responses to unrelated vaccine antigens when given along with them via the oral or nasal route. $^{78}$ The CT-B has been shown to be a potent immunomodulator besides its vector function for the toxic subunit A. It was recently shown that parenteral administration of purified CT-B, in the absence of autoantigen, delays the onset of diabetes in non-obese diabetic mice and that the administration of recombinant B subunit of $E$ coli heat-labile enterotoxin, a molecule structurally and functionally related to CT-B, prevents collagen induced arthritis. ${ }^{10}$ We have recently explored the immunological properties of recombinant CT-B (rCT-B) in a well characterised model of mucosal inflammation, the IL-12 driven, Thl cell mediated inflammation occurring in SJL/J mice after rectal administration of trinitrobenzene sulfonic acid (TNBS). ${ }^{11}$ We showed that oral administration of rCT-B inhibits the onset of TNBS induced colitis and reverses the established colitis.

Recent studies have focused on the molecular mechanisms underlying Th1 and Th2 cell development. ${ }^{12-14}$ Polarisation of naïve T cells into differentiated effector subsets is a tightly controlled process dependent on the activity of specific transcription factors. ${ }^{12}{ }^{15} 16$ STAT-1 and STAT-4 transcription factors are specifically associated with IFN- $\gamma$ and IL-12/IL-23 signaling in $\mathrm{T}$ lymphocytes and play a key role in regulating Thl cytokine production at the transcriptional level. ${ }^{12} 1718$ However, STAT-1 and STAT-4 deficient cells still retain the ability to produce some IFN- $\gamma$ thus suggesting the role of alternative signaling pathways in development and expansion of Thl cells. In this context, it was recently shown that full polarisation of Thl cells also requires the activity of the transcription factor T-bet. ${ }^{19}$ In contrast, optimal differentiation of Th2 cells strictly relies on the activity of STAT- $6 .^{20}$ In the present study we examined if rCT-B modulates mucosal Thl cell signaling. We show that oral administration of rCT-B in mice with TNBS induced colitis inhibits the activation of Thl associated transcription factors STAT-4, STAT-1, and

Abbreviations: $C D$, Crohn's disease; $C T$, cholera toxin; IBD, inflammatory bowel disease; LPMC, lamina propria mononuclear cells; TNBS, trinitrobenzene sulfonic acid; UC, ulcerative colitis. 
T-bet without affecting the expression of active STAT-6. Additionally, we provide evidence that rCT-B inhibits secretion of Thl cytokines and expression of Thl associated transcription factors in CD mucosal explants.

\section{MATERIALS AND METHODS \\ Production and purification of $\mathrm{rCT}-\mathrm{B}$}

The Vibrio Cholerae strain 0395-tacCTB, lacking the CT-A gene, was used as source to produce rCT-B (kindly supplied by $\mathrm{Dr} \mathrm{R}$ Rappuoli, Istituto Ricerche Immunobiologiche, Chiron, Siena, Italy). rCT-B was produced and purified as previously described ${ }^{11}$ according to the protocol described by Lebens et $a^{21}$ with minor modifications. All rCT-B preparations contained $<10 \mathrm{pg} / \mathrm{ml}$ LPS as determined by Quantitative Chromogenic Limulus Amebocyte Lysate test (QLC-1000, Biowhittaker, Walkersville, MD, USA).

\section{Induction of colitis and feeding of $\mathrm{rCT}-\mathrm{B}$}

Specific pathogen free 4-5 week old male SJL/J mice were obtained from Charles River (Calco, Italy) and maintained in a pathogen free environment animal facility. Animals were treated in accordance with the European Community guidelines and the local institute ethics committee. To induce TNBS-colitis, $3.75 \mathrm{mg}$ of TNBS (pH 1.5-2.0; Sigma Chemical Co, St Louis, MO, USA) in $150 \mu \mathrm{l}$ of a $50 \%$ ethanol solution was administered via rectum to lightly anaesthetised mice as previously described. ${ }^{11}$

In some experiments, $6 \mathrm{mg}$ of the haptenating agent, oxazolone (4-ethoxymethylene-2-phenyl-2-oxazolin-5-one, OXA) (Sigma Chemical Co) in $150 \mu$ l of $50 \%$ ethanol solution was administered intrarectally.

Mice, which received TNBS per rectum, were fed with $100 \mu \mathrm{g}$ of rCT-B in $500 \mu \mathrm{l}$ of $0.35 \mathrm{M} \mathrm{NaHCO}_{3}$ or $500 \mu \mathrm{l}$ of $0.35 \mathrm{M} \mathrm{NaHCO}_{3}$ at the time of TNBS instillations and every day, over a four day period, using an 18 gauge feeding needle. Mice treated with $50 \%$ ethanol intrarectally and which had received $500 \mu \mathrm{l}$ of $0.35 \mathrm{M} \mathrm{NaHCO}$ alone were used as controls for TNBS colitis.

\section{Isolation and culture of murine lamina propria mononuclear cells}

Lamina propria mononuclear cells (LPMC) were isolated from freshly obtained colonic specimens using a modification of the technique described by Van der Heijden and Stok ${ }^{22}$ as previously described. ${ }^{11}$ Cultures of murine LPMC were performed in complete medium consisting of RPMI 1640 supplemented with $2 \mathrm{mM}$ L-glutamine, $10 \mathrm{mM}$ HEPES buffer, $10 \mu \mathrm{g} / \mathrm{ml}$ gentamicin, $100 \mathrm{U} / \mathrm{ml}$ each of penicillin and streptomycin, and 10\% FCS (Hy-Clone). To measure the capacity of isolated LPMC to produce cytokines, the LPMC populations were cultured in complete medium at $1 \times 10^{6}$ cells $/ \mathrm{ml}$ in 48 -well plates (Costar, Corning Incorporated, NY, USA) coated or uncoated with anti-CD3 $\epsilon$ $\mathrm{Ab}$ (clone 145-2C11; Pharmingen, San Diego, CA, USA) as previously described. ${ }^{11}$ Cultures were also added with $1 \mu \mathrm{g} / \mathrm{ml}$ soluble CD28 Ab (clone 37.51; Pharmingen). For STAT-6 activation LPMC were stimulated with $10 \mathrm{ng} / \mathrm{ml}$ recombinant murine IL-4 for 30 minutes (Pharmingen). After 48 hour culture, culture supernatants were harvested and assessed for the presence of IFN- $\gamma$ by ELISA. To measure IL- 12 production, murine LPMC cells were preincubated for 18 hours with $1000 \mathrm{U} / \mathrm{ml}$ recombinant murine IFN- $\gamma$ (Genzyme-R\&D Systems, Abingdon, Oxon, UK) followed by stimulation with $0.03 \%$ Staphylococcus aureus Cowan strain I (SAC; Calbiochem, La Jolla, CA, USA) for an additional 24 hours. Cytokine concentrations were determined by commercially available specific ELISA kits as previously described. ${ }^{11}$

\section{Cell extracts from LPMC}

Whole cell extracts were prepared as previously described. ${ }^{23}$ Briefly, mouse LPMC were lysed in ice cold whole cell extraction buffer (20 mM Hepes pH 7.9, $50 \mathrm{mM} \mathrm{NaCl}, 0.5 \%$ NP-40, 1 mM DTT, 10 mM EDTA and 2 mM EGTA, $10 \mu \mathrm{g} / \mathrm{ml}$ leupeptin, $100 \mathrm{mM}$ sodium fluoride (NaF), $0.5 \mathrm{mM}$ PMSF, $10 \mathrm{mM}$ sodium orthovanadate and sodium molybdate). The lysate was incubated 30 minutes on a shaker at $4{ }^{\circ} \mathrm{C}$ and insoluble debris was removed by centrifugation $(10000 \mathrm{~g}$ at $4^{\circ} \mathrm{C}, 10$ minutes) and the lysate was stored at $-80^{\circ} \mathrm{C}$.

\section{Patients and samples}

Mucosal samples were taken from intestinal resection specimens of inflamed and non-inflamed regions of 11 patients (median age, 31 years; range 29-57) with moderate to severe CD undergoing surgery. In seven patients, the primary site of involvement of the disease was the terminal ileum; in the remaining four patients the disease involved the terminal ileum and the colon. Four patients were receiving corticosteroids at the time of resection. Indication for surgery was fibrostenosis in seven patients and a poorly responsive disease to medical therapy in four patients. Control samples included macroscopically and microscopically unaffected ileal areas from five patients undergoing bowel resection for right colon neoplasia. All the experiments were approved by the local ethics committee. Informed consent was obtained from all patients before collecting the samples.

\section{Human LPMC and tissue explant cultures and tissue extract preparations}

Intestinal mucosa was cut in small pieces $(2-3 \mathrm{~mm})$. Some of these pieces were immediately analysed, whereas the remaining were placed in 24-well plates in complete medium. Tissue specimens were incubated with or without the addition of rCT-B $(10 \mu \mathrm{g} / \mathrm{ml})$ at $37^{\circ} \mathrm{C}$ for four hours. Thereafter, mucosal samples were washed in complete medium to remove rCT-B and incubated for an additional 16-18 hours in complete medium. In addition, mucosal specimens from two CD patients were used to isolate LPMC. ${ }^{24}$ An aliquot of LPMC was incubated with or without rCT-B $(10 \mu \mathrm{g} / \mathrm{ml})$ at $37^{\circ} \mathrm{C}$ for four hours, then stimulated with IFN- $\gamma(200 \mathrm{U} / \mathrm{ml}$; Peprotech Inc Ltd, London, UK) for additional 14 hours, followed by $0.03 \%$ of SAC for a further 24 hours. At the end, culture supernatants were collected and analysed for IL- 12 production. The remaining LPMC were incubated with or without rCT-B $(10 \mu \mathrm{g} / \mathrm{ml})$ at $37^{\circ} \mathrm{C}$ for four hours and then stimulated with plate bound anti-CD $3 \mathrm{mAb}$ (OKT3) and soluble anti-CD28 mAb (clone 9.3; $1 \mu \mathrm{g} / \mathrm{ml}$, kindly provided by Professor Carl June, Cancer Center, University of Pennsylvania, Philadelphia, PA, USA) for a further 48 hours. Cell-free supernatants were then analysed for IFN- $\gamma$ production. $\mathrm{CD}^{+}$cells were also isolated from LPMC of one CD patient using anti-CD3 magnetic beads (Dynal Biotech Inc, Oslo, Norway). Purified $\mathrm{CD}^{+}{ }^{+}$cells were then either left untreated or treated with rCT-B for four hours and then directly stimulated according to the manufacturer's recommendation (Dynal Biotech Inc). IL-12 and IFN- $\gamma$ content was evaluated by specific ELISA kits (R\&D Systems).

Total proteins were prepared from mucosal samples using a Dounce tissue grinder (Wheaton Science Products, Millville, NJ, USA) in $0.5 \mathrm{ml}$ of lysis buffer containing $0.01 \mathrm{M}$ Hepes (pH 7.9), 1 mM EDTA, 6 mM KCl, 0.02\% NP40, 1 mM DTT, $1 \mathrm{mM} \mathrm{NaF}, 10 \mu \mathrm{g} / \mathrm{ml}$ aprotinin, $10 \mu \mathrm{g} / \mathrm{ml}$ leupeptin, and $1 \mathrm{mM}$ PMSF, and $1 \mathrm{mM} \mathrm{Na} \mathrm{VO}_{4}$ (all from Sigma Chemical Co). After cell lysis, samples were incubated on ice for 30 minutes and sedimented by centrifuging the lysates at $10000 \mathrm{~g}$ for 10 minutes $\left(4^{\circ} \mathrm{C}\right)$. 

Determination of phosphorylated STAT-4 level in
human mucosal samples

Total proteins $(500 \mu \mathrm{g} / \mathrm{sample})$ were incubated with antiSTAT-4 (C-20; Santa Cruz Biotechnology, Santa Cruz, CA, USA) in RIPA buffer ( $150 \mathrm{mM} \mathrm{NaCl}, 1 \%$ Nonidet P-40, 0.5\% sodium deoxycholate, and $0.1 \%$ SDS) containing a cocktail of protease and phosphatase inhibitors $(20 \mu \mathrm{g} / \mathrm{ml}$ aprotinin, $2 \mathrm{mM}$ PMSF, and $10 \mathrm{mM} \mathrm{Na}_{3} \mathrm{VO}_{4}$ ) at $4{ }^{\circ} \mathrm{C}$ for two hours. Immune complexes were collected by incubation with protein A/G agarose (Santa Cruz Biotechnology), washed three times with lysis buffer, and boiled for five minutes in sample buffer for SDS-PAGE. Immunoprecipitates from extracts containing the same amount of protein were analysed by western blotting with Ab against phosphotyrosine STAT4 (Y693) (Zymed Laboratories Inc, San Francisco, CA, USA) and subsequent incubation with HRP-conjugated goat antirabbit IgG (Calbiochem). The $\mathrm{Ab}$ reaction was detected with a chemiluminescence detection kit (Amersham International, Arlington Heights, IL, USA). After STAT-4 p-Tyr analysis, blots were stripped by incubation for 20 minutes at $50^{\circ} \mathrm{C}$ in stripping buffer ( $2 \%$ SDS, $62.5 \mathrm{mM}$ Tris- $\mathrm{HCl}$, and $100 \mathrm{mM} \mathrm{2-}$ $\mathrm{ME}$ ) and then incubated with Ab against STAT-4 (Santa Cruz Biotechnology).

\section{Western blot analysis}

Whole cell extracts $(30 \mu \mathrm{g})$ were separated by $7 \%$ or $10 \%$ SDS-PAGE gel and blotted onto nitrocellulose membranes. Blots were incubated with rabbit polyclonal Abs against STAT-1, STAT-4 and STAT-6 (Santa Cruz Biotechnology, Inc, E-23, C-20 and S-20, respectively) and reacted with antirabbit horseradish peroxidase (HRP) coupled secondary antibody (Calbiochem) using an ECL system (Amersham, Little Chalfont, UK). Tyrosine phosphorylated Abs against STAT-1 (Upstate, Lake Placid, NY, USA), STAT-4, and STAT-6 (Santa Cruz Biotechnology, Inc) were used to detect the phosphorylation status of these transcription factors. For the detection of T-bet in mice, total proteins ( $30 \mu \mathrm{g} / \mathrm{sample}$ ) were separated on a $10 \%$ SDS-PAGE gel. A commercially available monoclonal $\mathrm{Ab}$ against mouse T-bet (Santa Cruz Biotechnology, Inc), followed by a HRP-peroxidase conjugated goat antimouse IgG antibody (DAKO SpA, Milan, Italy) was used. The analysis of T-bet in human samples ( $100 \mu \mathrm{g}$ / sample) was performed using rabbit polyclonal anti T-bet Abs (a generous gift of Dr L H Glimcher, Department of Medicine, Harvard Medical School, Boston, MA, USA). After detection of T-bet, blots were stripped and incubated with a rabbit anti$\beta$-actin antibody (Sigma Chemical Co), followed by a goat antirabbit antibody conjugated to HRP.

\section{RNA and cDNA preparation and semiquantitative reverse transcriptase polymerase chain reactions} RNA was extracted from freshly mucosal samples with RNeasy Protect kit (Qiagen Inc, Valencia, CA, USA) according to the manufacturer's instructions. One $\mu \mathrm{g}$ of RNA was reverse transcribed in a final volume of $50 \mu \mathrm{l}$ containing $400 \mathrm{U}$ of murine leukemia virus reverse transcriptase (GibcoBRL Life Technologies Inc, Gaithersburg, MD, USA), 2 U of RNase Block (Roche), $0.5 \mathrm{mM}$ of a mix of dATP, dTTP, dCTP, dGTP (Gibco-BRL Life Technologies Inc), 10 mM DTT, $1 \mu \mathrm{M}$ of oligo dT and RT buffer according to the manufacturer's instructions. Reverse transcription was carried out at $37^{\circ} \mathrm{C}$ for 90 minutes. An equivalent amount of cDNA per sample was amplified using specific primers for p40 and p35 IL-12 subunits, IFN- $\gamma(10 \mu \mathrm{l} /$ reaction $)$ and GaPDH ( $3 \mu \mathrm{l} /$ reaction $)$. For the amplification of IL-12/p35 and IL-12/p40 transcripts the PCR reactions were performed by using specific primers from Stratagene (La Jolla, CA, USA) according to the manufacturer's instructions. IFN- $\gamma$ primers (M-Medical, Milan, Italy) were as follows: 5'-GCA TCG TTT TGG GTT
CTC TTG GCT GTT ACT GC-3' and 5'-GGT CGT CGA TTT TGT CCC TTC GCT TTT TCC TC-3'; GaPDH primers (M-Medical) were: 5'-GTC TTC ACC ATG GAG AAG GTC-3' and 5'-CAT GCC AGT GAG CTT CCC GTT CA-3'. PCR were performed in a total volume of $50 \mu \mathrm{l}$ as previously described. ${ }^{25}$ The level of RNA transcripts was measured by Fluor-S Multimager (BioRad Laboratories, Hercules, CA, USA) and expressed as arbitrary units.

\section{RESULTS}

Effect of rCT-B administration on IL- 12 and IFN- $\gamma$ production and signaling in mice with Th-1 mediated TNBS colitis

In initial studies we evaluated the effect of rCT-B administration on TNBS colitis course. rCT-B treated mice display a less sustained weight loss than in mice treated with TNBS alone (fig 1A). In addition, a reduction of IL-12 and IFN- $\gamma$ production by LPMC isolated from colons of mice treated with rCT-B was observed (fig IB), without affecting IL-4 production (not shown) in LPMC from mice with TNBS colitis.

As IL-12 signals through the STAT-4 pathway to promote IFN- $\gamma$ gene transcription, ${ }^{26}{ }^{27}$ we then investigated whether the rCT-B induced inhibition of IL-12 production was associated with changes in STAT-4 activation. For this purpose, total cell extracts were obtained from LPMC of mice with TNBS colitis treated or not with rCT-B. As shown in figure $2 \mathrm{~A}$, tyrosine phosphorylation of STAT-4 was clearly detected in cell extracts obtained from mice with TNBS colitis as compared to ethanol treated mice. However, treatment of mice with rCT-B caused a clear reduction in STAT-4 phosphorylation.

Recent studies have shown that optimal Thl cell response requires the activation of multiple transcription factors. Among these, T-bet appears to play a key role in driving mucosal Thl cytokine differentiation in experimental colitis. ${ }^{28}$ Therefore, we also examined the effect of treatment with rCTB both on T-bet expression and phosphorylation of STAT-1, one of its major inducer. As shown in figure 2A, STAT-1 phosphorylation and T-bet expression were barely detectable in ethanol treated mice. Induction of colitis associated with enhanced phosphorylation of STAT-1 and T-bet expression. However, the expression of these transcription factors was reduced by rCT-B (fig 2A). As reduction of T-bet expression enhances IL-4 signaling in LPMC, ${ }^{28}$ we next evaluated if the rCT-B mediated inhibition of T-bet associated with enhanced STAT-6 activation. As a positive control, we used total extracts from LPMC of mice with oxazolone (OXA) colitis, an experimental colitis mediated by IL-4-driven Th2 cells, ${ }^{29}$ either left untreated or treated in vitro for 30 minutes with IL-4 (OXA+IL-4). As expected, STAT-6 phosphorylation was evident in LPMC isolated from mice with OXA-mediated colitis, and those cells responded to the stimulation with exogenous IL- 4 by further enhancing the level of p-STAT- 6 (fig 2B). In contrast, no p-STAT-6 was seen in LPMC extracts from mice with TNBS colitis either left untreated or treated with rCT-B (fig 2B), indicating that rCT-B treatment does not stimulate IL-4 production.

\section{Effect of rCT-B treatment on IL- 12 expression and STAT-4 phosphorylation in organ culture of CD mucosal explants}

As TNBS colitis shares immunological similarities with CD, we then extended our analysis to the effects of rCT-B on the synthesis of Thl cytokines and expression of Thl associated transcription factors in mucosal explants from CD patients and non-IBD controls. As expected, transcripts for both IL-12 subunits were expressed at higher level in samples from involved mucosal area of CD patients than non-IBD controls 
A
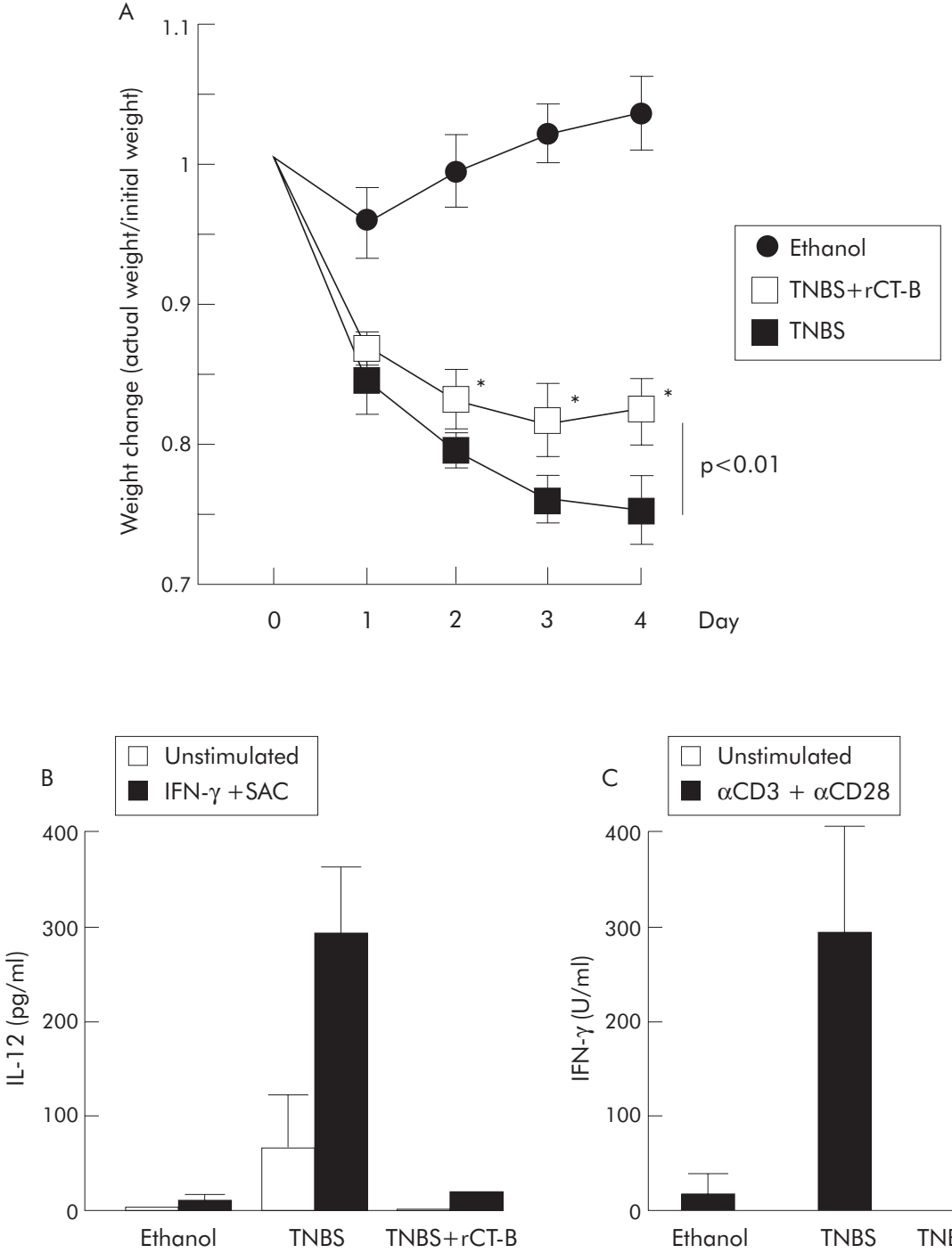

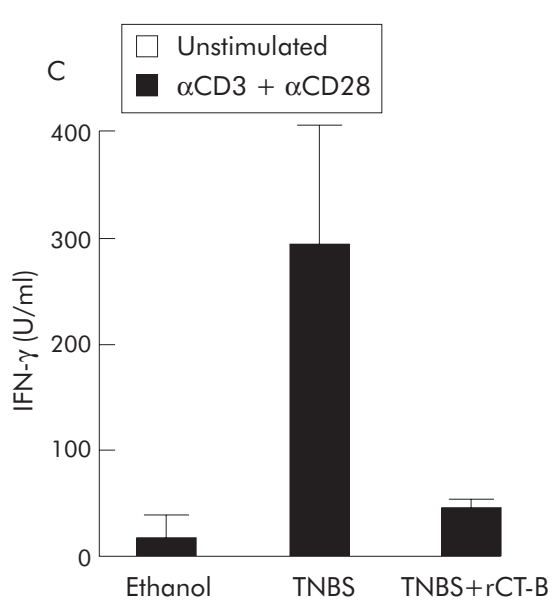

Figure 1 Oral administration of $\mathrm{rCT}-\mathrm{B}$ inhibits the induction of TNBS colitis and the mucosal production of IL-12 and

IFN- $\gamma$. SJL mice were treated by rectal instillation with TNBS to induce colitis or with $50 \%$ ethanol alone, as colitis controls. Mice were fed with $100 \mu \mathrm{g}$ rCT-B in $0.35 \mathrm{M} \mathrm{NaHCO}_{3}$ or with $0.35 \mathrm{M} \mathrm{NaHCO}_{3}$ alone as described in material and methods. (A)

Determination of variations in body weight. Each point represents the cumulative mean weight data from five different experiments. In all experiments, each group consisted of at least five mice. Bars represent standard errors. ${ }^{*} p<0.01$ mice with TNBS induced colitis and concomitant administration of $\mathrm{rCT}-\mathrm{B} / \mathrm{NaHCO}_{3}$ versus mice with TNBS induced colitis and concomitant administration of $\mathrm{NaHCO}_{3}$. (B) The production of IL-12 and IFN- $\gamma$ was measured in the culture supernatants of LPMC isolated from the colon of mice with TNBS colitis treated or not with oral $\mathrm{rCT}-\mathrm{B}$, or from the colon of mice treated with $50 \%$ ethanol alone. To enhance IL-12 production, LPMC were treated with SAC and IFN- $\gamma$ as indicated in materials and methods. For IFN- $\gamma$ production, LPMC cultures were performed in the absence of any stimulant or in the presence of antiCD3/CD28. Data indicate mean from two independent experiments. In each experiment, cultures of pooled LPMC extracted from the colon of five mice per group were performed. Bars indicate standard error.
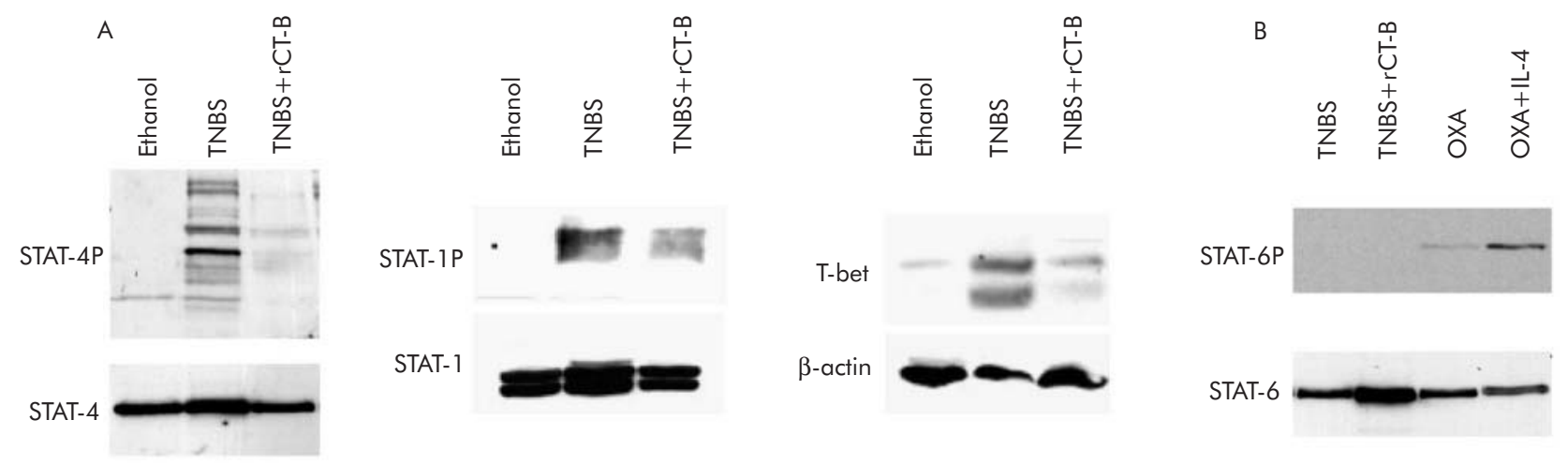

Figure 2 rCT-B administration inhibits IL-12 and IFN- $\gamma$ signaling in mice with TNBS colitis. Total cell extracts were prepared from LPMC of mice sacrificed four days affer TNBS instillation. Mice with TNBS colitis were either left untreated (TNBS) or treated with oral $r C T-B$ (TNBS+rCT-B). As control of TNBS treatment, mice were instilled with 50\% ethanol alone (Ethanol). One representative experiment of three independent experiments is shown. (A) Thirty $\mu \mathrm{g}$ of whole cell lysates were separated on a 7\% SDS-PAGE, transferred to a nitrocellulose membrane and blotted sequentially with the indicated antibodies to evaluate both phosphorylation status and total content of STAT molecules. For the analysis of T-bet, $100 \mu \mathrm{g}$ of whole cell lysates were subjected to immunoblotting with anti T-bet antibodies. After detection of T-bet, blots were stripped and incubated with a mouse anti $\beta$-actin antibody to ascertain equivalent loading of the lanes. (B) LPMC total extracts were obtained from mice with TNBS or OXA colitis either left untreated or treated with oral rCT-B (TNBS+rCT-B). Where indicated, LPMC were treated with IL-4 for 30 minutes. Thirty $\mu \mathrm{g}$ of whole cell lysates were subjected to immunoblot analysis with the indicated antibodies to evaluate both STAT-6 phosphorylation status and protein content. 
(fig 3A, B). Importantly, rCT-B treatment led to a marked inhibition in the expression of both p35 and p40 transcripts (fig 3A, B). These findings were also confirmed by the measurement of IL-12 p70 released into the supernatants of mucosal explant cultures (fig 3C). We also showed that expression of p-STAT-4 was more pronounced in tissue extracts from inflamed areas of $\mathrm{CD}$ in comparison to that seen in uninvolved areas from the same patients and nonIBD controls (fig 3D). Consistent with this, rCT-B reduced the level of p-Stat-4 in inflamed mucosal tissues from CD (fig 3D).

\section{Effect of rCT-B treatment on IFN- $\gamma$ expression and signaling in ex vivo $C D$ mucosal samples}

As IL-12 mediated STAT-4 activation promotes the development of IFN- $\gamma$ producing Th cells, we investigated the effect of rCT-B on expression and signaling of IFN- $\gamma$ in CD mucosal explants. As shown in figure $4 \mathrm{~A}-\mathrm{C}$, expression of IFN- $\gamma$ RNA transcripts as well as the IFN- $\gamma$ secretion were upregulated in $\mathrm{CD}$ mucosal samples, and they were markedly inhibited by rCT-B treatment. In agreement with this, rCT-B also reduced the expression of intracellular transcription factors that are associated with IFN- $\gamma$ signaling, such as p-STAT- 1 and T-bet (fig 4D) P-STAT-6 was barely detectable both in CD and nonIBD mucosal explants regardless of whether tissue was left untreated or treated with rCT-B (not shown).

\section{Effect of rCT-B treatment on IL- 12 and IFN- $\gamma$ production by purified LPMC isolated from CD mucosal explants}

To examine whether rCT-B directly affects the production of cytokines by intestinal LPMC, cells were isolated from two $\mathrm{CD}$ tissue specimens and incubated with rCT-B before the addition of stimuli that are known to enhance either IL-12 or
IFN- $\gamma$ production. As shown in table 1 , stimulation of CD LPMC with IFN- $\gamma$ and SAC enhanced the secretion of the biologically active heterodimeric IL-12p70, and this production was reduced by nearly $60 \%$ by rCT-B. Similarly, the synthesis of IFN- $\gamma$ induced in CD LPMC by anti-CD3/CD28 was reduced by rCT-B (table 1 ). In a separate experiment we also examined whether rCT-B modulated IFN- $\gamma$ production by purified $\mathrm{CD}^{+}$lymphocytes. Treatment of $\mathrm{CD}^{+}$cells with rCT-B reduced IFN- $\gamma$ secretion ( $10.1 \vee 43.2 \mathrm{pg} / \mathrm{ml})$.

\section{DISCUSSION}

There is no doubt that polarisation of naïve T cells toward the Thl type requires the combined action of multiple cytokines and activation of specific signaling pathways. While IL-12 driven STAT-4 signaling appears to be sufficient to induce IFN $-\gamma$ gene transcription and trigger Thl cell differentiation, activation of STAT-l and induction of T-bet play a decisive role in expanding and sustaining Thl mucosal cell responses. Consistent with this, high expression of active STAT-4 and Tbet has been documented both in CD tissue and TNBS induced colitis. ${ }^{28}{ }^{30-33}$ In the present study, we demonstrate that oral administration of rCT-B inhibits IL-12 and IFN- $\gamma$ production and suppresses Thl associated transcription factors in the gut of mice with TNBS colitis. Therefore, these data confirm and expand results of our previous work which showed the ability of rCT-B to prevent or cure TNBS colitis in mice. In addition in this study, the effects of rCT-B on CD mucosal explants and LPMC isolated from CD tissue specimen were also examined showing that rCT-B reduced IL- 12 and IFN- $\gamma$ production and, in turn, the Thl cell signaling.

The molecular mechanism by which rCT-B limits the ongoing Thl mucosal inflammation in mice with TNBS colitis remains unclear. It is unlikely that rCT-B suppresses Thl cell response by promoting Th2 cell differentiation, given that no
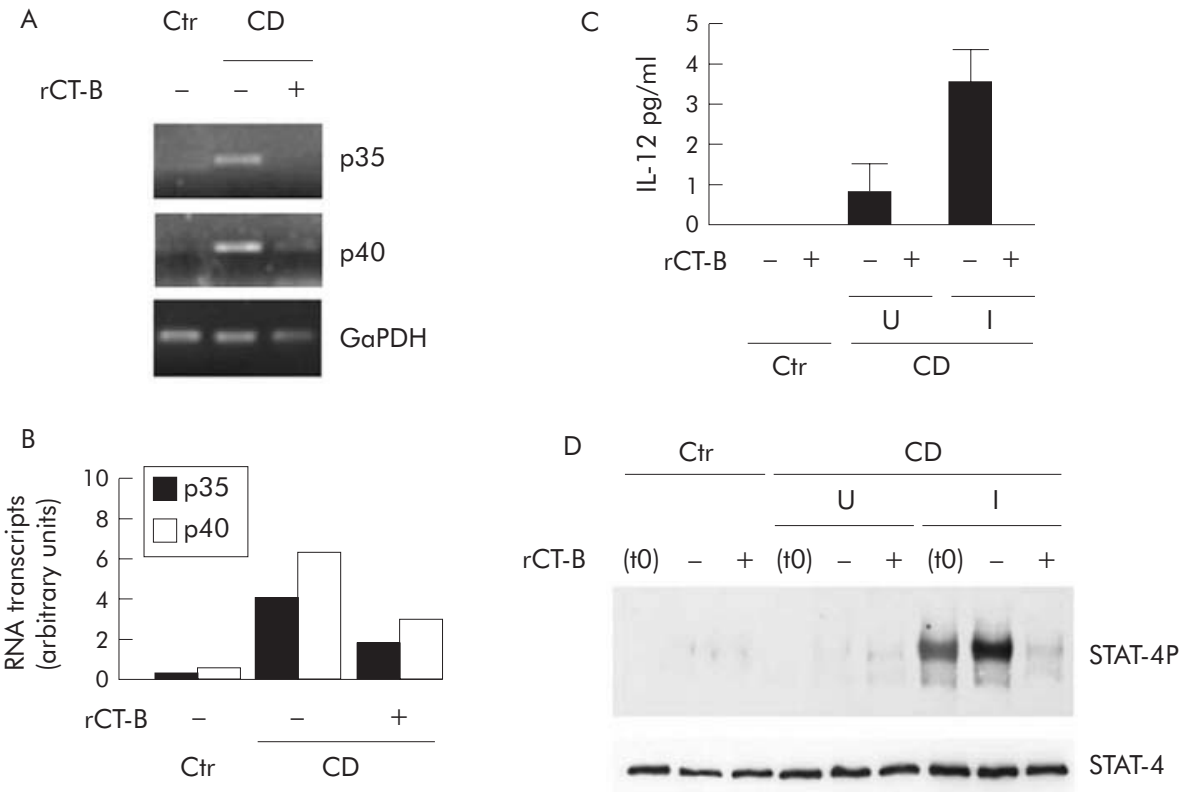

Figure 3 Effect of rCT-B treatment on IL-12 expression and STAT-4 phosphorylation in ex vivo mucosal explants. Mucosal samples from inflamed (I) and uninflamed (U) tissues of a CD patient and from a non-IBD control (Ctr). TO represents mucosal samples processed immediately as control for the basal ex vivo level and activation of the studied transcription factors. Where indicated, mucosal samples were treated for four hours with $\mathrm{rCT}-\mathrm{B}$ (10 $\mu \mathrm{g} /$ $\mathrm{ml}$ ). Thereafter, mucosal samples were washed in complete medium to remove $\mathrm{rCT}-\mathrm{B}$ and incubated for additional 16-18 hours in complete medium. One representative experiment of three independent experiments is shown. (A) RNA transcripts for IL-12 p35, p40 and GaPDH were analysed in mucosal tissue omogenates by semiquantitative PCR. (B) The levels of RNA stranscripts were measured by the Fluor-S Multimager and the relative values are expressed in arbitrary units. (C) Determination of IL-12 p70 in supernatants of cultured mucosal explants. The results shown are the mean (standard error) of values obtained in three separated experiments. (D) Total proteins (500 $\mu \mathrm{g} / \mathrm{sample}$ ) were immunoprecipitated with anti-STAT-4, and immunoblotted with anti-phosphotyrosine STAT-4 (Y693). After STAT-4 p-Tyr analysis, blots were stripped and then incubated with Ab against STAT-4. 
A

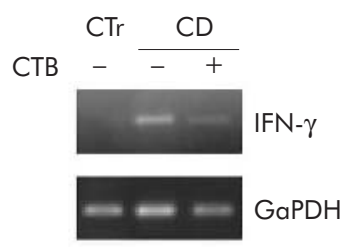

B

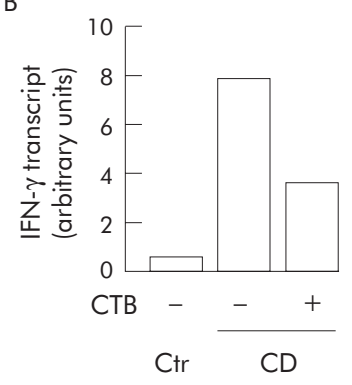

C

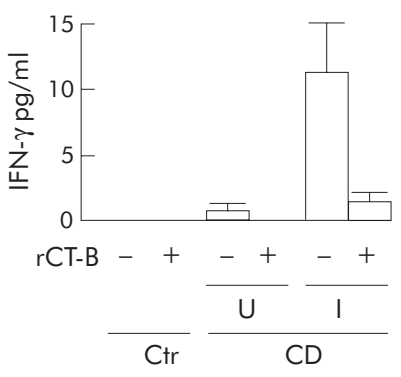

D

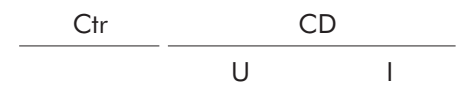

rCT-B

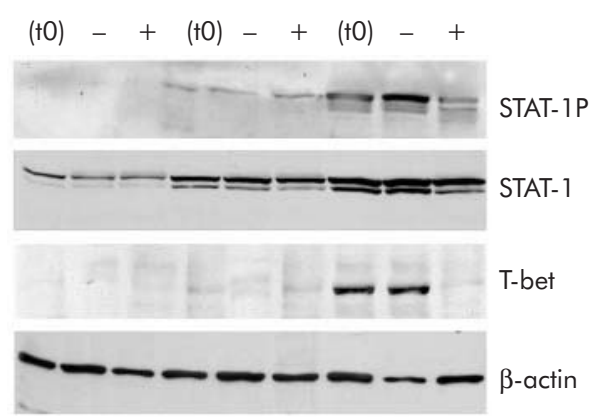

Figure 4 Effect of $\mathrm{rCT}$-B treatment on IFN- $\gamma$ signaling and expression in ex vivo $C D$ mucosal samples. Mucosal samples were taken from inflamed (I) and uninflamed (U) tissues of a CD patient or from a non-IBD control (Ctr). One representative experiment of three independent experiments is shown. (A) RNA transcripts for IFN- $\gamma$ and GaPDH were analysed in mucosal tissue omogenates by semiquantitative PCR.

(B) The levels of RNA transcripts were measured by Fluor-S Multimager and the relative values are expressed in arbitrary units. (C) Determination of IFN- $\gamma$ in supernatants of cultured mucosal explants. The results shown are the mean (standard error) of values obtained in three separated experiments. (D) Thirty $\mu \mathrm{g}$ of whole cell lysates were subjected to immunoblot analysis with the indicated antibodies to evaluate both total and phosphorylated STAT-1. TO represents mucosal samples processed immediately as control for the basal ex vivo content of the protein. Where indicated, mucosal samples were treated for four hours with rCT-B $(10 \mu \mathrm{g} / \mathrm{ml})$. Thereafter, mucosal samples were washed in complete medium to remove $\mathrm{rCT}-\mathrm{B}$ and incubated for additional 16-18 hours in complete medium. Total proteins $(100 \mu \mathrm{g} /$ sample) were immunoblotted with anti T-bet. After T-bet analysis, the level of $\beta$-actin was evaluated as an internal loading control. change in IL-4 synthesis and p-STAT-6 activation was seen in the colon of mice treated with rCT-B. A possibility is that rCT$\mathrm{B}$ favours the development and/or activity of regulatory $\mathrm{T}$ cells which would, in turn, dampen the local Thl cell response. This would be supported by the demonstration that administration of rCT-B can induce tolerance in the peripheral $\mathrm{T}$ cell compartment and protect animals against several immune mediated experimental diseases by inducing suppressive regulatory $\mathrm{T}$ cells. In this context, for example, it has been shown that CT-B promotes the induction of both IL10 and transforming growth factor- $\beta$ secreting $\mathrm{T}$ cells and synergises with low doses of LPS in stimulating IL-10 production by immature murine dendritic cells. ${ }^{34} 35$ However, it is noteworthy that in our experimental model, rCT-B administration did not increase mucosal production of IL- $10,{ }^{11}$ thus arguing against a primary role of IL- 10 in the negative regulation of TNBS colitis by rCT-B.

Pentameric B subunit of CT binds to GMl gangliosides on the membrane of all nucleated cells. In association with sphingomyelin and cholesterol, GMl is concentrate in caveolae-like structures of lymphocytes and in caveolae of

\begin{tabular}{|c|c|c|}
\hline & IL-12 & IFN- $\gamma$ \\
\hline $\begin{array}{l}\text { Unstimulated } \\
\text { SAC+IFN- } \gamma \\
\text { SAC+IFN- } \gamma+C T B \\
\alpha C D 3+\alpha C D 28 \\
\alpha C D 3+\alpha C D 28+C T B\end{array}$ & $\begin{array}{l}<0.5 \\
32(16) \\
12.4(11)\end{array}$ & $\begin{array}{l}<8 \\
60(20) \\
37(33)\end{array}$ \\
\hline
\end{tabular}

LPMC (200 000 cells in $200 \mu l)$ were cultured as specified in material and methods. Supernatants were collected and analysed by ELISA. The results represent the mean (standard error) of two separate experiments. The values are expressed in $\mathrm{pg} / \mathrm{ml}$. other cells that contain a high concentration of signaling molecules. ${ }^{36}{ }^{37}$ It is possible that binding of CT-B to GMI ganglioside receptors on IL-12 producing cells and T cells per se can trigger/modify signaling events which, in turn, may affect the function of target cells leading also to a reduced production of Thl inducing cytokines. This hypothesis is supported by the recent evidence that a mutant CT-B subunit, that does not bind GMl, fails to modulate leucocyte function. ${ }^{38}$ How the binding of the B subunit to GMl can trigger/influence signal transduction is not known. One possibility is that the binding of CT-B to GMl might influence the activity of integral membrane proteins that participate in cell signaling. Whatever the underlying mechanism, we believe that the beneficial effect of oral administration of rCT-B on the Thl mucosal inflammation is not purely dependent on the ability of rCT-B to enhance tolerance in the gut associated lymphoid tissues. In fact, in the present study we show that the expression of IL-12 and IFN- $\gamma$ as well as the activation of Thl transcription factors are dramatically inhibited by in vitro treatment of CD mucosal explants with rCT-B. Importantly, the expression of phosphorylated but not total STAT-1 and -4 was suppressed by rCT-B, clearly indicating that the rCT-B mediated inhibition of Thl associated transcription factors in $\mathrm{CD}$ is not secondary to a toxic effect of rCT-B. Our data also suggest the possibility that the rCT-B-induced changes in the activation of such Thl transcription factors are secondary to the ability of rCT-B to suppress directly the synthesis of IL-12 and IFN- $\gamma$. Indeed, we observed a reduction of IL-12 production by isolated LPMC pre-incubated with rCT-B and then stimulated with SAC, a known IL-12 inducer. Interestingly, we were also able to demonstrate that rCT-B impairs IFN- $\gamma$ synthesis by purified $\mathrm{CD}^{+}$cells from $\mathrm{CD}$ tissue specimen in the absence of IL-12-producing cells. At the moment we cannot distinguish whether the inhibitory effect on IFN- $\gamma$ production is due to a direct action of rCT-B on isolated $\mathrm{CD}^{+}$cells or 
whether is a consequence of an apoptotic process induced by rCT-B as previously demonstrated. ${ }^{39} 40$

In recent years evidence showing that IL-12/IL-12R signals contribute to the local inflammatory response in $\mathrm{CD}$ has been accumulated. Indeed, increased expression of IL-12 and IL$12 \mathrm{R}$ correlates with high activation of STAT- 4 and IFN- $\gamma$ production in the inflamed mucosa of CD patients. ${ }^{30} 41$ Evidence has also been provided to suggest that IFN- $\gamma$, in turn, contributes to expand the local Thl differentiation by signaling through the STAT-1 pathway and enhancing the transcription of T-bet. ${ }^{19} 8^{31-33}$ Consistent with this, it was recently demonstrated that T-bet is expressed in LPMC in CD but not in UC and control patients, and that overexpression of T-bet results in an earlier onset of Thl mediated colitis. ${ }^{28}$ Additionally, it was reported that T-bet deficient $\mathrm{T}$ cells fail to induce Thl mediated colitis in an adoptive transfer model of colitis, while IL-4 mediated colitis is enhanced in T-bet deficient mice. Therapeutic interventions aimed at interfering with Thl cell development and/or perpetuation could therefore be useful in limiting the intestinal inflammation in patients with CD. This is also strongly supported by the recent demonstration that administration of neutralising IL-12 antibodies in CD patients is followed by a significant reduction in the clinical activity. ${ }^{42}$ Therefore, the demonstration that rCT-B dramatically inhibits the activation of Thl transcription factors and Thl cytokine production suggests the possibility that oral administration of rCT-B to CD patients represents a useful means to achieve clinical remission or amelioration of the disease.

\section{ACKNOWLEDGEMENTS}

The authors are grateful to Dr Rino Rappuoli (IRIS, Chiron SpA Siena, Italy) for the kind gift of Vibrio cholerae 0395-tacCT-B and Dr Laurie Glimcher (Department of Medicine, Harvard Medical School, Boston, MA, USA) for providing antihuman T-bet antibody. A special thank you to Corrado Volpe for the culture of the Vibrio cholerae, to N Di Carlo for animal care, and to E Morassi for preparing drawings.

\section{Authors' affiliations \\ E M Coccia, M E Remoli, C Di Giacinto, B Del Zotto, E Giacomini,}

M Boirivant, Department of Infectious, Parasitic and Immune-mediated Diseases, Istituto Superiore di Sanità, Rome, Italy

G Monteleone, Cattedra di Gastroenterologia, Dipartimento di Medicina Interna, University Tor Vergata di Roma, Rome, Italy

This work was supported by grants from the Istituto Superiore di Sanità (99F and $2 A E I$ to $E C$ and to $M B$ ).

Competing interests: none declared.

\section{REFERENCES}

1 Podolsky D. Inflammatory bowel disease. N Engl J Med 2002;347:417-29.

2 Duchmann R, Kaiser I, Hermann E, et al. Tolerance exists towards resident intestinal flora but is broken in active inflammatory bowel disease (IBD). Clin Exp Immunol 1995; 102:448-55.

3 Fais S, Capobianchi M, Pallone F, et al. Spontaneous release of interferon gamma by intestinal lamina propria lymphocytes in Crohn's disease: kinetics of in vitro response to interferon gamma inducers. Gut 1991;32:403-7.

4 Fuss I, Neurath $M$, Boirivant $M$, et al. Disparate CD4+ lamina propria (LP) lymphokine secretion profiles in inflammatory bowel disease. Crohn's disease LP cells manifest increased secretion of IFN-gamma, whereas ulcerative colitis LP cells manifest increased secretion of IL-5. J Immunol 1996;157:1261-70.

5 Parronchi $\mathbf{P}$, Romagnani $\mathrm{P}$, Annunziato F, et al. Type $1 \mathrm{~T}$-helper cell predominance and IL-12 expression in the gut of patients with Crohn's disease. Am J Pathol 1997;150:823-32.

6 Monteleone G, Biancone L, Marasco R, et al. Interleukin 12 is expressed and actively released by Crohn's disease intestinal lamina propria mononuclear cells. Gastroenterology 1997;1 12:1169-78.

7 Elson CO. Cholera toxin and its subunits as potential oral adjuvants. Curr Top Microbiol Immunol 1989; 146:29-33.

8 Lycke $\mathrm{N}$. The mechanism of cholera toxin adjuvanticity. Res Immunol 1997; 148:504-20.

9 Williams N, Stasiuk L, Nashar T, et al. Prevention of autoimmune disease due to lymphocyte modulation by the B-subunit of Escherichia coli heat-labile enterotoxin. Proc Natl Acad Sci U S A 1997;94:5290-5.
10 Sobel D, Yankelevich B, Goyal D, et al. The B-subunit of cholera toxin induces immunoregulatory cells and prevents diabetes in the NOD mouse. Diabetes 1998;47:186-91

11 Boirivant M, Fuss IJ, Ferroni L, et al. Oral administration of recombinant cholera toxin subunit $B$ inhibits IL-12-mediated murine experimental (trinitrobenzene sulfonic acid) colitis. J Immunol 2001;166:3522-32.

12 Rengarajan J, Szabo S, Glimcher L. Transcriptional regulation of Th1/Th2 polarization. Immunol Today 2000;21:479-83.

13 Leonard W. STATs and cytokine specificity. Nat Med 1996;2:968-9

14 Agnello D, lankford C, Bream J, et al. Cytokines and transcription factors that regulate Thelper cell differentiation: new players and new insights. J Cli Immunol 2003;23:147-61.

15 Grogan J, Mohrs M, Harmon B, et al. Early transcription and silencing of cytokine genes underlie polarization of T helper cell subsets. Immunity $2001 ; 14: 205-15$

16 Szabo S, Sullivan B, Peng S, et al. Molecular mechanisms regulating Th1 immune responses. Annu Rev Immunol 2003;21:713-58.

17 O'Shea J. Jaks, STATs, cytokine signal transduction, and immunoregulation: are we there yet? Immunity 1997;7:1-11

18 Oppmann B, Lesley R, Blom B, et al. Novel p19 protein engages IL-12p40 to form a cytokine, IL-23, with biological activities similar as well as distinct from IL-12. Immunity 2000;13:715-25.

19 Szabo S, Kim S, Costa G, et al. A novel transcription factor, T-bet, directs Th1 lineage commitment. Cell 2000; 100:655-69.

20 Kurata $\mathrm{H}$, Lee $\mathrm{H}, \mathrm{O}^{\prime}$ Garra $\mathrm{A}$, et al. Ectopic expression of activated Stat6 induces the expression of Th2-specific cytokines and transcription factors in developing Th1 cells. Immunity 1999;11:677-88

21 Lebens M, Johansson S, Osek J, et al. Large-scale production of Vibrio cholerae toxin $B$ subunit for use in oral vaccines. Biotechnology 1993;11:1574-8.

22 Heijden PVd, Stok W. Improved procedure for the isolation of functionally active lymphoid cells from the murine intestine. J Immunol Methods 1987; 103:161-7.

23 Remoli ME, Giacomini E, Lutfalla G, et al. Selective expression of type I IFN genes in human dendritic cells infected with Mycobacterium tuberculosis. J Immunol 2002; 169:366-74.

24 Bull DM, Bookman MA. Isolation and functional characterization of human intestinal mucosal lymphoid cells. J Clin Invest 1977;59:966-74.

25 Quaranta M, Camponeschi B, Straface E, et al. Induction of interleukin-15 production by HIV-1 nef protein: a role in the proliferation of uninfected cells. Exp Cell Res 1999;250:112-21.

26 Nishikomori R, Usui T, Wu C, et al. Activated STAT4 has an essential role in Th1 differentiation and proliferation that is independent of its role in the maintenance of IL-12R beta 2 chain expression and signaling. J Immunol 2002; 169:4388-98.

27 Thierfelder W, Deursen Jv, Yamamoto K, et al. Requirement for Stat4 in interleukin-12-mediated responses of natural killer and T cells. Nature 1996;382:171-4

28 Neurath $M$, Weigmann B, Finotto S, et al. The transcription factor T-bet regulates mucosal T cell activation in experimental colitis and Crohn's disease. $J$ Exp Med 2002;195:1129-43.

29 Boirivant M, Fuss IJ, Chu A, et al. Oxazolone colitis: A murine model of T helper cell type 2 colitis treatable with antibodies to interleukin 4. J Exp Med 1998;188:1929-39.

30 Parrello T, Monteleone G, Cucchiara S, et al. Up-regulation of the IL-12 receptor beta 2 chain in Crohn's disease. J Immunol 2000;165:7234-9.

31 Heath VL, Kurata H, Lee HJ, et al. Checkpoints in the regulation of T helper 1 responses. Curr Top Microbiol Immunol 2002;266:23-39.

32 Neurath MF, Finotto S, Glimcher LH. The role of Th1/Th2 polarization in mucosal immunity. Nat Med 2002;8:567-73.

33 Matsuoka K, Inove N, Sato T, et al. T-bet upregulation and subsequen interleukin 12 stimulation are essential for induction of Th1 mediated immunopathology in Crohn's disease. Gut 2004;53:1303-8.

34 Lavelle EC, McNeela E, Armstrong ME, et al. Cholera toxin promotes the induction of regulatory $T$ cells specific for bystander antigens by modulating dendritic cell activation. J Immunol 2003;171:2384-92.

35 Sun J, Xiao B, Lindblad M, et al. Oral administration of cholera toxin B subunit conjugated to myelin basic protein protects against experimental autoimmune encephalomyelitis by inducing transforming growth factor-beta-secreting cells and suppressing chemokine expression. Int Immunol 2000;12:1449-57.

36 Parton RG. Ultrastructural localization of gangliosides; GM1 is concentrated in caveolae. J Histochem Cytochem 1994;42:155-66.

37 Simons K, lkonen E. Functional rafts in cell membranes. Nature 1997;387:569-72.

38 Aman AT, Fraser S, Merritt EA, et al. A mutant cholera toxin B subunit that binds GM1-ganglioside but lacks immunomodulatory or toxic activity. Proc Natl Acad Sci U S A 2001;98:8536-41.

39 Wang M, Bregenholt S, Petersen JS. The cholera toxin B subunit directly costimulates antigen-primed CD4+ T cells ex vivo. Scand J Immunol 2003;58:342-9.

40 Yankelevich B, Soldatenkov VA, Hodgson J, et al. Differential induction of programmed cell death in CD8+ and CD4+ T cells by the B subunit of cholera toxin. Cell Immunol 1996; 168:229-34.

41 Mudter J, Neurath MF. The role of signal transducers and activators of transcription in T inflammatory bowel diseases. Inflamm Bowel Dis 2003:9:332-7.

42 Mannon PJ, Fuss IJ, Mayer L, et al. Anti-interleukin-12 antibody for active crohn's disease. N Engl J Med 2004;351:2069-79. 